\title{
Comparative Investigation of Alternative Negative Staining Reagents in Bacterial Morphological Study
}

\author{
Masaaki Minami ${ }^{*}$, Hiroshi Takase ${ }^{2}$ \\ ${ }^{1}$ Department of Bacteriology, Nagoya City University, Nagoya, Japan \\ ${ }^{2}$ Core Laboratory, Graduate School of Medical Sciences, Nagoya City University, Nagoya, Japan \\ Email:^minami@med.nagoya-cu.ac.jp
}

How to cite this paper: Minami, M. and Takase, H. (2017) Comparative Investigation of Alternative Negative Staining Reagents in Bacterial Morphological Study. Journal of Biosciences and Medicines, 5, 17-24.

https://doi.org/10.4236/jbm.2017.510002

Received: September 15, 2017

Accepted: October 20, 2017

Published: October 23, 2017

Copyright $\odot 2017$ by authors and Scientific Research Publishing Inc. This work is licensed under the Creative Commons Attribution International License (CC BY 4.0).

http://creativecommons.org/licenses/by/4.0/

\begin{abstract}
Negative staining is an effective method that can be used for electron microscopic study to observe fine structural morphology without destruction of bacterial structure. Although uranium acetate is used worldwide as a general dyeing solution, it is extremely difficult to use it by a new purchase at a research institution because it falls under the nuclear regulation substance in Japan. Therefore, we examined alternative reagents for negative staining that could replace uranium acetate through bacterial observation with an electron microscope. Escherichia coli, Pseudomonas aeruginosa, Staphylococcus aureus, and Streptococcus pyogenes were examined by four stain reagents (phosphotungstic acid (PTA), EMstainer, TI blue, and uranium acetate). Pre cultured bacteria were stained with each stain reagents on a copper grid, washed with PBS, and observed with a transmission electron microscope. In the comparison between bacterial structures, the cell wall structure and bacterial flagella could be observed well in the order of PTA, EMstainer, and uranium acetate. With TI blue staining, flagella could be observed very poorly. In comparison between bacteria, gram negative bacteria such as Escherichia coli and Pseudomonas aeruginosa, could be observed well as compared with gram positive cocci such as Staphylococcus aureus and Streptococcus pyogenes. The uranium acetate looked very coarse in background particles. Since crystals tend to precipitate, TI blue also required filtering, and electron beams were absorbed by the agglomerated crystals, and the frequency of electronic burning occurred high frequency. In this study, there was clear difference in the observation conditions depending on the type of bacteria and the kind of the staining reagents. Especially, it was confirmed that good negative staining features of Pseudomonas aeruginosa by electron microscope were obtained by PTA and EMstainer staining. These alternative reagents are considered to be a candidate for a negative staining.
\end{abstract}




\section{Keywords}

Negative Staining, Electron Microscope, Bacterial Micromorphology, Phosphotungstic Acid, EMstainer

\section{Introduction}

Bacteriological investigation categorizes morphological and functional observation, respectively. In the morphological observation, there is an observation with an optical microscope and an electron microscope. Usually, it is necessary to fix the cells in order not to change the structure of the cells either by optical microscope or electron microscope. However, with this fixed operation, the cell structure may be changed, which may indicate another form. In order to minimize the change of features, there is a negative staining method [1]. Although this method cannot observe the internal structure of cells by ultrathin sections, it is possible to observe the surface structure of flagella and cells with the closest conditions to the shape of living cells [2] [3].

In the electron microscope staining method that also includes negative staining, it is common worldwide to use uranium acetate [4] [5]. Since it is extremely difficult to purchase new uranium acetate at a research institution because it falls under the nuclear regulation substance in Japan, it is preferable to use other reagents instead of uranium acetate for electron microscopy. In the electron microscope reagent, TI blue (formerly Pt-blue) and EMstainer are commercially available in Japan. These reagents were developed in Japan, respectively [6]. Although TI blue and EMstainer may be derived from platinum and gadolinium, respectively, detailed information on the product details has been disclosed precisely. Although these reagents are considered useful for observing ultrathin sections, there are few reports reported for negative staining [7]. Furthermore, little study of negative staining using these staining solutions for bacteria has been made [8] [9]. As an alternative dyeing solution optimal for negative staining has not been established yet, establishment of a staining reagent for electron microscope except uranyl acetate is desired.

In this study, we evaluated the effectiveness of negative staining on bacterial observation using phosphotungstate acid (PTA), EMstainer, TI blue, and uranium acetate.

\section{Material and Methods}

\subsection{Bacteria and Reagents}

Clinical isolates of Pseudomonas aeruginosa (P. aeruginosa), Escherichia coli (E. coli), Staphylococcus aureus (S. aureus), and Streptococcus pyogenes (S. pyogenes) were used in this study. Phosphotungstate acid (PTA) was purchased from Kanto chemical co. Inc. (Tokyo, Japan). EMstainer, and TI blue were purchased from Nisshin EM (Tokyo, Japan). Uranium acetate preserved at Nagoya 
City University was used in this study. The observation concentrations of PTA, uranyl acetate, EM, and TI blue were $0.4 \%$. After $2 \%$ PTA solution initially was adjusted to $\mathrm{pH} 7.0$ by addition of $0.1 \mathrm{~mol} / \mathrm{L} \mathrm{KOH}$, the PTA solution was readjusted to $0.4 \%$ by addition of $\mathrm{RO}$ water when using.

\subsection{Negative Staining Procedure}

A fresh bacterial colony was cultured overnight on TSAII sheep blood agar (Nihon Becton Dickinson, Tokyo, Japan) at $37^{\circ} \mathrm{C}$. The bacterial cell was centrifuged and suspended in $1 \mathrm{~mL}$ of PBS ( $\mathrm{pH}$ 7.0.).

This mixture $(50 \mu \mathrm{L})$ was placed on a sheet of parafilm, and 400-mesh formvar-carbon-coated copper grid F-400 (Nisshin EM, Tokyo, Japan) was floated for 30 seconds on the drops. After the grid was rinsed on drops of PBS, the parts of the needless specimen was wiped. Then, the grid was staining immediately with drop of PTA, uranyl acetate, EMstainer, TI blue for 30 seconds, respectively. It was drawn off from the edge of the grid with filter paper. The grid was directly placed into grid box and was allowed it air-dry for several minutes before observation. The copper grid F-400 was placed on a regular holder and imaged on a JEM-1011J microscope (JEOL Ltd, Tokyo, Japan) operated at $80 \mathrm{kV}$. Images were recorded on a Bio Scan Camera (GatanCCD camera) (Roper Technologies, Inc., Sarasota, FL 34240, USA). Ten fields for each sample were randomly photographed.

\section{Result}

Gram negative bacteria (E. coli and P. aeruginosa) and Gram positive bacteria ( $S$. aureus and $S$. pyogenes) were examined by negative staining. These bacteria were representative microorganisms of high importance both morphologically and clinically microbiologically. Four staining reagents (PTA, EMstainer, TI blue, and uranium acetate,) were used.

The structures of the cell wall and flagellation were better in order of PTA, EMstainer, uranium acetate. In EMstainer staining, it appeared clearly at a concentration of $0.4 \%$. The number of flagellum with use of EMstainer were highest visible among four staining reagents. TI blue had to be filtered before dying, it was impossible to observe because crystals were precipitated. Also, the outline of the surface of the bacteria blurred, and it looked ambiguous. It was totally invisible as a whole on the internal structure. In TI blue staining, aggregation was likely to occur, electron beams were absorbed by this aggregate, and electron beam burning occurred frequently. The background particles appeared very rough in uranium acetate. With TI blue staining, the surroundings of the bacteria and the surface of the background were whitened out.

In E. coli, flagellum could not be confirmed in TI blue (Figure 1). In P. aeruginosa, flagellum was not also clearly visible in TI blue (Figure 2). The structure in the flagella was most clearly visible in PTA. In the PTA, the cell wall inside the bacteria was seen most clearly. In $S$. aureus, the contour of the surface was 


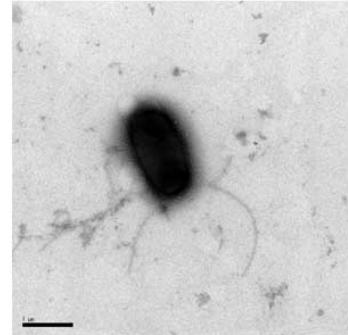

(a)

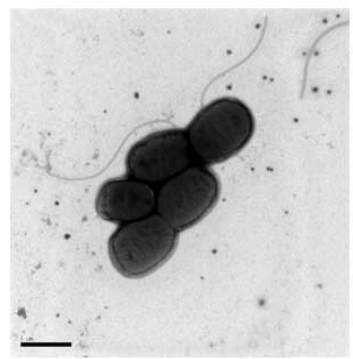

(c)

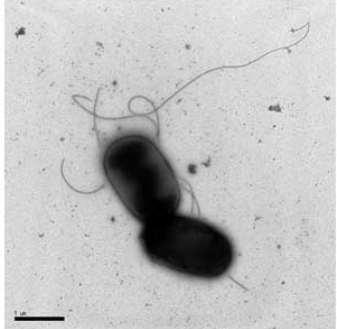

(b)

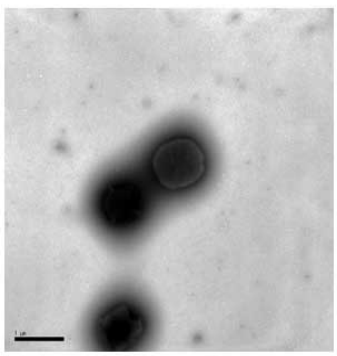

(d)

Figure 1. Morphological pattern of Escherichia coli observed by electron microscope by various staining methods. (a) EM stainer, (b) uranium acetate, (c) PTA, (d) TI blue. Scale bar: $1 \mu \mathrm{m}$.

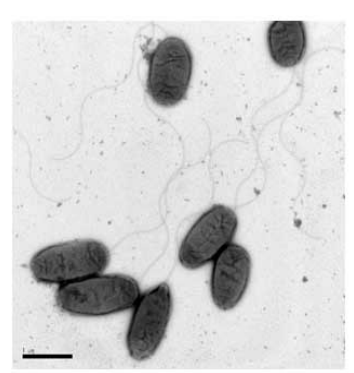

(a)

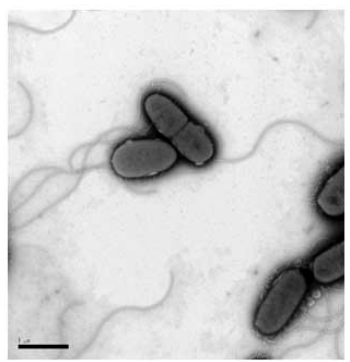

(c)

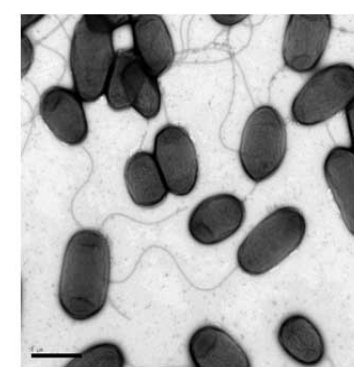

(b)

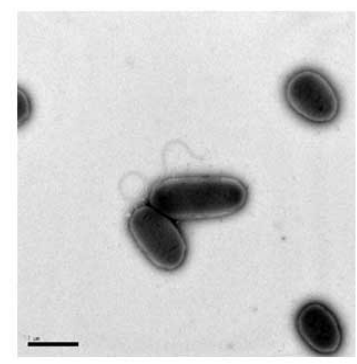

(d)

Figure 2. Morphological pattern of Pseudomonas aeruginosa observed by electron microscope by various staining methods. (a) EM stainer, (b) uranium acetate, (c) PTA, (d) TI blue. Scale bar: $1 \mu \mathrm{m}$.

blurred in the EMstainer, and it looked ambiguous (Figure 3). The cell wall structure looked destructive was in uranium acetate. The structure within the 


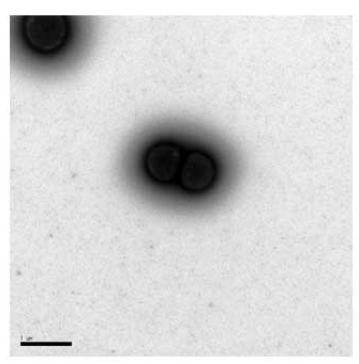

(a)

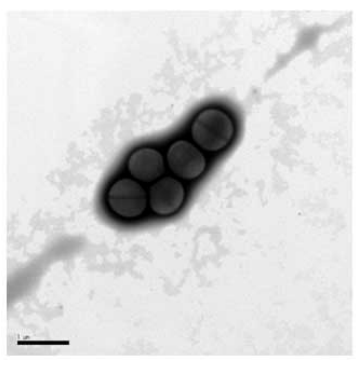

(c)

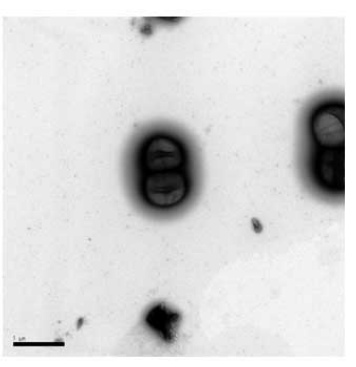

(b)

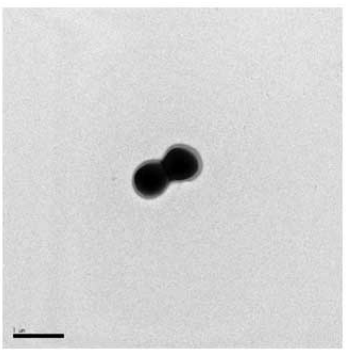

(d)

Figure 3. Morphological pattern of Staphylococcus aureus observed by electron microscope by various staining methods. (a) EM stainer, (b) uranium acetate, (c) PTA, (d) TI blue. Scale bar: $1 \mu \mathrm{m}$.

cell was not clearly visible in uranium acetate and TI blue. Also, with uranium acetate, the bacterial body was rapidly broken compared with other staining. EMstainer and uranium acetate showed fine particles in the background. The size of bacterial cell was shrinking in TI blue. In S. pyogenes, the contours of the surface were blurred and appeared vague in uranium acetate and TI blue (Figure 4). Septal wall between the bacteria was seen with PTA. In EMstainer, a lot of fine particles were seen in the background. In comparison between bacteria, Gram negative bacteria such as, E. coli and $P$. aeruginosa could be observed better as compared with Gram positive cocci such as $S$. aureus and $S$. pyogrnes. With TI blue staining, flagella could not be observed very clearly in both $E$. coli and $P$. aeruginosa.

\section{Discussion}

Negative staining is a simple and rapid method to study the morphology and the structure of particulates specimens. It allows the determination of shapes at the molecular level with high-resolution electron [1]. The negative staining method is based on the principle that there is no reaction between the staining reagents and the samples. On drying, the electron-dense metal atoms envelop the samples. The differences between the samples and the surrounding heavy metal atoms with respect to their density produce the necessary contrast. The sample appears to be light surrounded by a dark background of dried stain. The electron beam passes the low electron density of the samples, but not through the metallic background. The structure is inferred from the distribution that the sample 


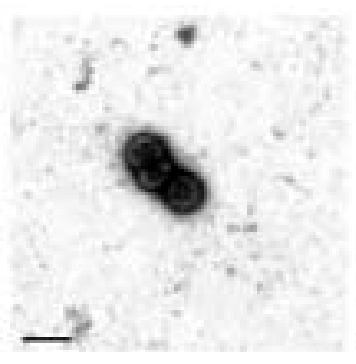

(a)

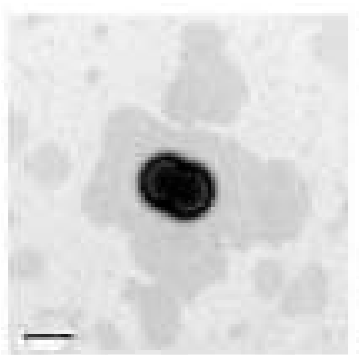

(c)

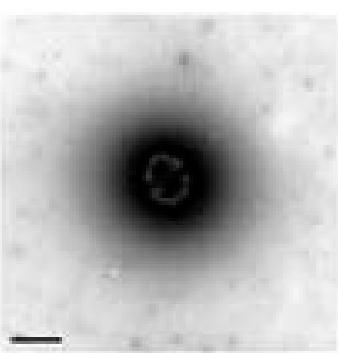

(b)

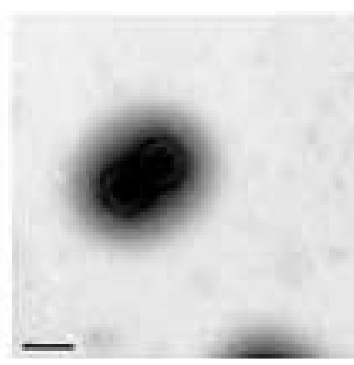

(d)

Figure 4. Morphological pattern of Streptococcus pyogenes observed by electron microscope by various staining methods. (a) EM stainer, (b) uranium acetate, (c) PTA, (d) TI blue Scale bar: $1 \mu \mathrm{m}$.

imposes on the stain. The clarity of sample detail depends on the degree to which the strain remains amorphous as it dries as well as on the thickness of the dried negative stain envelope [2].

Many factors affect the appearance of negatively stained samples. The shape and size of samples are influenced by both the mode of negative stain application and the reagents itself. The $\mathrm{pH}$ and concentration of the reagents, concentration of the samples, temperature, duration of staining, the nature of support film, and the length of time it takes for the samples to dry on the support film influence the final image of the samples [2].

The selection of staining reagents is depended on both the objective of the investigation and the type of samples. A negative staining may give excellent results with one sample but totally unsuccessful results with another type of sample. When a sample is negatively stained for the first time, this may become a serious limitation [1].

Usually optical microscope analysis is convenient for bacterial investigation. In the optical microscope observation, only the spherical or rod-shapes are confirmed. If the objective bacteria are small growing, they cannot be seen. Thus, the additional time for culturing time is needed. Direct electron microscopy based on negative staining is the most rapid method for the examination and the identification of bacteria [4]. Treatment and prognosis of cases depend on the rapid and correct bacterial diagnosis. This is possible with direct electron microscopy because bacteria cell have characteristic morphologies; that is, shape, size, and ultrastructure. However, within a group, individual bacteria are difficult 
to differentiate on the basis of appearance alone. Direct electron microscopy can be accomplished within minutes or hours. It can visualize any bacteria type present, and previous knowledge of the type of bacteria is not necessary. From this point, electron microscope observation may be superior to the optical microscope observation with respect to bacterial analysis.

Our study demonstrated that $P$. aeruginosa was easily visible. It may be related to the chemical interaction between cell surface structure and secretion and negative staining solution. In fact, unlike $E$. coli, $S$. aureus and $S$. pyogenes, $P$. aeruginosa secretes a large amount of mucin outside the cells [5]. This mucin may be useful for establishing contrast with the staining solution.

Uranyl acetate and PTA are the most commonly used negative staining. Uranyl acetate acts as a fixative at the $\mathrm{pH}$ it is used for negative staining [10]. It stabilizes lipids and minimizes the deleterious effect of drying on cells organelles [11]. Effects of various negative staining on cell wall morphology of gram-negative bacteria indicate that in $2 \%$ uranyl acetate, the cell surface is mostly smooth and there is little stain penetration into the cell wall [5]. Thus we also conducted this experiment under these previous conditions.

Unlike uranyl acetate, PTA does not have affixation effect and may favor the dissociation of the quaternary protein structure into smaller units [2]. It lacks a stabilizing effect on membranes and mitochondria and may damage them. It shows relatively low negative contrast compared with that generated by uranyl acetate [11]. However, observation of bacteria does not take a long time and is not fixed by PTA. As there is no denaturation of bacterial cells, there is an advantage that it can be observed as it is.

TI blue is a general term for a polymeric compound of deep blue, green, or purple in which Pt is coordinate with amide groups. It is an aqueous solution prepared from the reaction of cis-Pt and thymidine [12]. In the previous study, researchers evaluated the effect of TIblue as a flagellum from $E$. coli stained with TI blue showed that TIblue is potentially useful in negative staining situations [8]. However, in our study, it was very difficult to adjust the conditions of use of TI blue, and bacterial flagella could not be seen clearly with TI blue staining.

The injury by the electron beam in the white part inside the bacteria was easily occurred with use of EMstainer and TI blue by negative staining. Adjusting the concentration is important because the white part increases as the dyeing density increases. As, TI blue also required filtering because crystals tend to precipitate. Electron beams were absorbed by the agglomerated crystals, and the frequency of electronic burning in the observation field of view was high. Frequent washing after staining was essential for EMstainer and TI blue. Especially, TI blue seemed to be less likely to become the first selective reagent for negative staining as ready-made products due to condition setting and resolution instability.

In this study, there was a clear difference in the observation state depending on the type of bacteria and the kind of the staining solution. Especially, it was confirmed that good negative staining features of $P$. aeruginosa by electron 
microscope were obtained by PTA and EMstainer staining. These alternative reagents are considered to be a candidate for a negative staining.

\section{Acknowledgements}

We thank Mr. Masashi Ishihara and Ms. Miwako Fujimura for special encouragement. This study was supported by a grant-in-aid for research from the Nagoya City University, Japan.

\section{References}

[1] Hayat, M.A. and Miller, S.E. (1990) Negative Staining. McGraw-Hill Publishing Company, New York.

[2] Baschong, W. and Aubi, U. (2006) Chapter 23. Negative Staining. In: Celis, J.E., Ed., Cell Biology: A Laboratory Handbook, 3rd Edition, Academic Press, Cambridge, MA, 233-240.

[3] Mörgelin, M. (2017) Negative Staining and Transmission Electron Microscopy of Bacterial Surface Structures. Bacterial Pathogenesis, 1535, 211-217. https://doi.org/10.1007/978-1-4939-6673-8_13

[4] Laue, M. and Bannert, N. (2010) Detection Limit of Negative Staining Electron Microscopy for the Diagnosis of Bioterrorism-Related Microorganisms. Journal of Applied Microbiology, 109, 1159-1168. https://doi.org/10.1111/j.1365-2672.2010.04737.x

[5] Ramphal, R. and Arora, S.K. (2001) Recognition of Mucin Components by Pseudomonas aeruginosa. Glycoconjugate Journal, 18, 709-713. https://doi.org/10.1023/A:1020823406840

[6] Tanaka, K. and Inagaki, K. (1993) Enhancement of the BSE Signal from Hydrous SEM Samples by Use of a Platinum Blue. Journal of Electron Microscopy, 42, 255.

[7] Wells, B., Horne, R.W., Lund, B.M. and King, N.R. (1983) The Ultrastructure of Pseudomonas avenae. I. Paracrystalline Surface layer and Extracellular Material. Micron, 14, 11-28.

[8] Ichinose, A. and Wada, A. (2007) Negative-Staining Improvement of the GramNegative Bacteria by Phosphotungstic Acid and Ammonium Molybdate. Journal of Electron Microscopy Technology for Medicine and Technology, 21, 1-6.

[9] Inaga, S., Katsumoto, T., Tanaka, K., Kameie, T., Nakane, H. and Naguro, T. (2007) Platinum Blue as an Alternative to Uranyl Acetate for Staining in Transmission Electron Microscopy. Archives of Histology and Cytology, 70, 43-49. https://doi.org/10.1679/aohc.70.43

[10] Yamaguchi, K., Suzuki, K., and Tanaka, K. (2010) Examination of electron stains as a substitute for uranyl acetate for the ultrathin sections of bacterial cells. Journal of Electron Microscopy (Tokyo), 59, 113-118. https://doi.org/10.1093/jmicro/dfp045

[11] Nakakoshi, M., Nishioka, H. and Katayama, E. (2011) New Versatile Staining Reagents for Biological Transmission Electron Microscopy That Substitute for Uranyl Acetate. Journal of Electron Microscopy (Tokyo), 60, 401-407. https://doi.org/10.1093/jmicro/dfr084

[12] Yusuf, M., Millas, A.L., Estandarte, A.K., Bhella, G.K., McKean, R., Bittencourt, E. and Robinson, I.K. (2014) Platinum Blue Staining of Cells Grown in Electrospun Scaffolds. Biotechniques, 57, 137-141. https://doi.org/10.2144/000114206 